\title{
NEW EQUIAFFINE CHARACTERIZATIONS OF THE ELLIPSOIDS RELATED TO AN EQUIAFFINE INTEGRAL INEQUALITY ON HYPEROVALOIDS
}

\author{
ZEJUn Hu AND CHENG XING*
}

\begin{abstract}
In this paper, we study hyperovaloids from the perspective of the equiaffine differential geometry. As the main result, we establish an optimal integral inequality of the hyperovaloids in terms of the normalized affine scalar curvature and the squared norm of the equiaffine Weingarten form. Since the integral inequality becomes an integral equality if and only if the hyperovaloids are equiaffinely equivalent to the ellipsoids, our results give new equiaffine characterizations of the ellipsoids.
\end{abstract}

Mathematics subject classification (2010): 53A15, 53C20, 53C24.

Keywords and phrases: Ellipsoid, hyperovaloid, integral inequality, equiaffine characterization, rigidity theorem, semi-parallel cubic form.

\section{REFERENCES}

[1] B. ANDREws, Contraction of convex hypersurfaces by their affine normal, J. Differ. Geom. 43 (1996), 207-230.

[2] L. J. Alias, A. G. Colares, A further characterization of ellipsoids, Results Math. 48 (2005), 1-8.

[3] M. ANTIĆ, H. LI, L. VRANCKEN, X. WANG, Affine hypersurfaces with constant sectional curvature, Pacific J. Math. 310 (2021), 275-302.

[4] W. BLASCHKE, Über affine Geometrie I: Isoperimetrische Eigenschaften von Ellipse und Ellipsoid, Ber. Verh. Sächs. Akad. Leipzig, Math.-Phys. Kl. 68 (1916), 217-239.

[5] W. BlaschKe, Vorlesungen über Differentialgeometrie, II, Springer, Berlin, 1923.

[6] E. CALABI, An extension of E. Hopf's maximum principle with an application to Riemannian Geometry, Duke Math. J. 25 (1958), 45-56.

[7] X. Cheng, Z. HU, On the isolation phenomena of locally conformally flat manifolds with constant scalar curvature - submanifolds versions, J. Math. Anal. Appl. 464 (2018), 1147-1157.

[8] X. Cheng, Z. Hu, A.-M. LI, H. LI, On the isolation phenomena of Einstein manifoldssubmanifolds versions, Proc. Am. Math. Soc., 146 (2018), 1731-1740.

[9] X. Cheng, Z. Hu, L. VRAnCKen, Every centroaffine Tchebychev hyperovaloid is ellipsoid, arXiv:1911.05222v1.

[10] X. Cheng, Z. Hu, Z. Yao, A rigidity theorem for centroaffine Chebyshev hyperovaloids, Colloq. Math. 157 (2019), 133-141.

[11] S. Y. Cheng, S. T. YAU, Complete affine hyperspheres, Part I. The completeness of affine metrics, Comm. Pure Appl. Math. 39 (1986), 17-30.

[12] A. DEICKE, Über die Finsler-Räume mit $A_{i}=0$, Arch. Math. 4 (1953), 45-51.

[13] F. Dillen, A. Martinez, F. Milan, F. G. Santos, L. Vrancken, On the Pick invariant, the affine mean curvature and the Gauss curvature of affine surfaces, Results Math. 20 (1991), 622-642.

[14] Z. HU, H. LI, L. VRANCKEN, Locally strongly convex affine hypersurfaces with parallel cubic form, J. Differ. Geom. 87 (2011), 239-307.

[15] Z. Hu, H. Li, U. Simon, L. VRancken, On locally strongly convex affine hypersurfaces with parallel cubic form. Part I, Differ. Geom. Appl. 27 (2009), 188-205. 
[16] C. C. Hsiung, J. K. Shahin, Affine differential geometry of closed hypersurfaces, Proc. London Math. Soc. 17 (1967), 715-735.

[17] J. Hu, G. XIONG, D. Zou, Affine isoperimetric inequalities for intersection mean ellipsoids, Calc. Var. Partial Diff. Equat. 58 (2019), Art. 191, pp. 20.

[18] M. Kozlows KI, U. Simon, Hyperfächen mit äquiaffiner Einsteinmetrik, Mathematica, Festschrift E. Mohr, TU Berlin, (1985), 179-190.

[19] A. LI, Q. HuAnG, D. XI, New sine ellipsoids and related volume inequalities, Adv. Math. 353 (2019), 281-311.

[20] A.-M. Li, U. Simon, G. S. Zhao, Z. Hu, Global Affine Differential Geometry of Hypersurfaces, 2nd ed., de Gruyter Expositions in Mathematics 11, Walter de Gruyter, Berlin/Boston, 2015.

[21] A.-M. LI, Some theorems in affine differential geometry, Acta Math. Sinica N. S. 5 (1989), 345-354.

[22] A.-M. LI, A characterization of ellipsoids, Results Math. 20 (1991), 657-659.

[23] E. LutwaK, Selected affine isoperimetric inequalities, A survey article, In: P. M. Gruber and J. M. Wills (eds.), Handbook Convex Geometry, pp. 153-176, North Holland, Amsterdam, 1993.

[24] E. LutwaK, D. Yang, G. Zhang, Orlicz projection bodies, Adv. Math. 223 (2010), 220-242.

[25] E. Lutwak, D. YAng, G. Zhang, Orlicz centroid bodies, J. Differ. Geom. 84 (2010), 365-387.

[26] M. Magid, P. Ryan, Flat affine spheres in $\mathbf{R}^{3}$, Geom. Dedicata 33 (1990), 277-288.

[27] K. Nomizu, T. Sasaki, Affine Differential Geometry, Cambridge University Press, Cambridge, (1994).

[28] A. V. Pogorelov, Complete affine minimal hypersurfaces, Soviet Math. Dokl. 38 (1989), 217-219.

[29] L. SANTALÓ, An affine invariant for convex bodies of $n$-dimensional space (Spanish), Portugal. Math. 8 (1949), 155-161.

[30] R. SCHNEIDER, Zur affinen Differentialgeometrie im Grossen, I, Math. Z. 101 (1967), 375-406.

[31] R. SCHNEIDER, Convex bodies: the Brunn-Minkowski theory, Encyclopedia of Mathematics and Its Applications, 151, Cambridge University Press, Cambridge, (2014).

[32] T. SASAKI, On affine isoperimetric inequality for a strongly convex closed hypersurface in the unimodular affine space $A^{n+1}$, Kumamoto J. Sci. (Math.) 16 (1984), 23-38.

[33] U. Simon, Minkowskische Integralformeln und ihre Anwendungen in der Differentialgeometrie im Grossen, Math. Ann. 173 (1967), 307-321.

[34] U. Simon, The Pick invariant in equiaffine differential geometry, Abh. Math. Sem. Univ. Hamburg 53 (1983), 225-228.

[35] U. Simon, Hypersurfaces in equiaffine differential geometry, Geom. Dedicata 17 (1984), 157-168.

[36] W. Süss, Zur relativen Differentialgeometrie V: Über Eihyperflächen im $\mathbf{R}^{n+1}$, Tohoku Math. J. 31 (1929), 202-209.

[37] U. Simon, A. Schwenk-Schellschmidt, H. Viesel, Introduction to the Affine Differential Geometry of Hypersurfaces, Lecture Notes, Science University Tokyo, 1991.

[38] L. VRANCKEn, Affine surfaces with higher order parallel cubic form, Tohoku Math. J. 43 (1991), $127-139$.

[39] L. VRANCKEN, Affine quasi umbilical hypersurfaces which are flat with respect to the affine metric, Results Math. 20 (1991) 756-776.

[40] L. VRAncken, A.-M. Li, U. Simon, Affine spheres with constant sectional curvature, Math. Z. 206 (1991), 651-658. 